\section{Mehr Effizienz durch Apps \& Co im Praxisalltag}

\author{
Bei einer Fortbildungsveranstaltung der Deutschen Apotheker- und Ärzte- \\ bank Anfang April in Magdeburg gab es für die Teilnehmer eine Orientierung, \\ wie die Nutzung moderner Medien den Praxisalltag verändern könnte.
}

$\mathrm{R}_{\mathrm{w}}^{\mathrm{u}}$ und 400.000 medizinische Apps weltweit soll es mittlerweile geben. Die von Experten genannten Zahlen wirken in ihrer enormen Dimension fast willkürlich gewählt. Doch für Ärzte und ihre Patienten ist eine andere Frage entscheidend: Welche Apps können für Ärzte und/oder Patienten tatsächlich hilfreich sein? Professor Christian Schmidt, Ärztlicher Direktor und Vorstand der Universitätsklinik Rostock, brachte während einer Fortbildungsveranstaltung im April im Magdeburger Haus der Heilberufe etwas Licht ins Dunkel.

Dass Patienten in den USA ihre Patientenakten in Apps herunterladen oder Mediziner in "Ärzte-Communities“ konkrete Fälle, teilweise anhand von Fotos oder Röntgenbildern, diskutieren, mag dort normal sein. Im Land der unbegrenzten Möglichkeiten schicke mittlerweile schließlich auch schon so mancher Kühlschrank seine Bestellung an den Supermarkt, wenn die Milch ausgegangen ist. Aber im Datenschützerland Deutschland? „Kann uns dieser ganze digitale Kram wirklich helfen?" Mit dieser provokanten Frage nahm Schmidt einigen kritisch eingestellten Teilnehmern an der von der Deutschen Apothekerund Ärztebank (apoBank) organisierten Fortbildung gleich den Wind aus den Segeln. Eingeladen hatte die apoBank zum Thema „Digital Health in der Medizin. Apps und Wearables im Alltag“ Ärzte, Zahnärzte und Psychotherapeuten sowie Apotheker und Veterinärmediziner. Kooperationspartner der Veranstaltung waren der Verband der leitenden Krankenhausärzte Deutschlands und die Ärztekammer Sachsen-Anhalt.

\section{Digitalisierung bei \\ unaufhaltsamer Mehrarbeit}

Sicher sei, so Referent Professor Schmidt, dass die Digitalisierung um Ärzte keinen Bogen machen wird. In den kommenden Jahren werden immer weniger Menschen in der Versorgung immer mehr
Leistungen erbringen müssen - auch in der Medizin. Der steigenden Zahl an Rentnern stehen immer weniger junge Ärzte gegenüber. Viele von ihnen bevorzugten Teilanstellungen in Krankenhäusern und Praxen. Wie ist die dadurch anfallende Mehrarbeit zu bewältigen? Schmidt ist überzeugt: „Produktivitätszuwachs lässt sich nur durch Digitalisierung gewährleisten.“

\section{Mit der richtigen App zu Interdisziplinarität}

Die richtigen Apps könnten Brücke zu gelebter Interdisziplinarität sein. Dazu gehörten zum Beispiel Apple-Apps wie "Clinicalkey“, „Health Manager“, „Medical Research“ oder „Workflow Management":

_Clinicalkey beispielsweise erlaube einen schnellen Zugriff auf einen großen Pool evidenzbasierter klinischer Informationen zu Diagnostik und Behandlung.

_Der Health Manager dagegen helfe Patienten, eigene Gesundheitswerte $\mathrm{zu}$ erfassen und im Blick zu behalten.

-Medical Research wiederum „sammelt" Patientendaten für Wissenschaftler in der medizinischen Forschung.

_Durch Workflow-Management-Apps könnten chronisch Kranke in Verbindung mit digitalen Endgeräten (Blut- druckmessgerät, EKG, Ergometer, Waage etc.) besser überwacht werden. Das sei eine Arbeitserleichterung für Ärzte und zugleich ein Weg zu gleichberechtigter, spezialisierter Versorgung in Stadt und Land, betonte Schmidt. Probleme sieht er noch in der Auswertung der Datenflut. Dank immer intelligenter werdender Systeme sei jedoch absehbar, dass künftig lediglich abweichende Werte angezeigt werden.

Apps, davon ist der Ärztliche Direktor überzeugt, werden mehr und mehr zu täglichen Begleitern. So könnten Compliance Tools bestimmte Patientengruppen an die Einnahme ihrer Medikamente, Bewegung oder auch Blutdruckmessungen erinnern. „Sowohl Ärzte als auch Patienten müssen für sich herausfinden, was für sie konkret Sinn macht und was nicht." Helfend könnten hier KVen durch die Entwicklung eigener Apps einspringen. „Aber die tun sich sehr schwer damit."

\section{Noch mehr Robotik wird kommen}

Wie Apps in den Alltag, so werden auch immer mehr OP-Roboter in Kliniken einziehen. „Kann ein Arzt 500 Eingriffe eines bestimmten Verfahrens vorweisen, greift der Roboter auf Erfahrungen von 80.000 und mehr zurück “, so der Universitätsmediziner. Der Anfang sei gemacht: Robotik stehe bereits heute für schonendere Operationen, sichere Biopsien oder genauere Ergebnisse beim Fräsen künstlicher Hüften. Schmidt: „Ob wir es wahrhaben wollen oder nicht: Der schwächste Punkt in der Kette ist bei manchen Tätigkeiten tatsächlich der Mensch."

Petra Zieler

\section{BIBB-Bericht 2017: Ausbildung in Praxen ist beliebt}

Wie schon in den Jahren zuvor, wurde auch 2016 ein Drittel aller neuen Ausbildungsverträge in nur zehn Berufen abgeschlossen. Dabei zeigen sich die Arztpraxen in Deutschland als durchaus attraktive Arbeitgeber: Sie erreichen immerhin mit 15.822 neu abgeschlossenen Verträgen mit angehenden Medizinischen Fachangestellten (MFA) Rang 6. Das geht aus dem aktuellen Berufsbildungsbericht 2017 hervor, den das Bundeskabinett vor Kurzem in Berlin verabschiedet hat. Nur auf weibliche Auszubildende bezogen, erreicht die Ausbildung zur MFA sogar Rang 2 - 15.465 der Neuverträge wurden nämlich mit jungen Frauen abgeschlossen. Erhoben werden die Daten vom Bundesinstitut für Berufsbildung (BIBB), in den aktuellen Report sind mehr als 300 Ausbildungsberufe eingegangen (Stichtag 30. September 2016).

Rebekka Höhl mit Material der dpa 